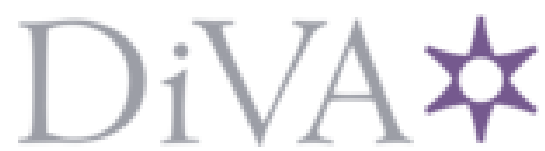

http://www.diva-portal.org

\title{
Postprint
}

This is the accepted version of a chapter published in Wiley Encyclopedia of Electrical and Electronics Engineering.

Citation for the original published chapter:

Larsson, T. (2017)

Telecommunication Exchange Evolution.

In: Webster, John G. (ed.), Wiley Encyclopedia of Electrical and Electronics Engineering (pp.

1-16). New York: John Wiley \& Sons

https://doi.org/10.1002/047134608X.W2043.pub2

N.B. When citing this work, cite the original published chapter.

Permanent link to this version:

http://urn.kb.se/resolve?urn=urn:nbn:se:hh:diva-34763 


\section{TELECOMMUNICATION EXCHANGE EVOLUTION}

\section{INTRODUCTION}

The telecommunications network is one of the most important systems created by humans in modern civilization. It enables people to communicate between continents at all times of the year and allows thoughts and ideas to be exchanged between families, companies, and governments. Several phone calls have changed history. The network also saves lives every day. An example is when mobile subscribers in their cars, or even the cars themselves, make calls to emergency numbers when part of or witnessing a traffic accident.

Today, due to its digitalization, the telecommunication network has been connected to converge and integrate with data and media distribution networks, and thus the Internet. Wireless connectivity enabled smart mobile phones to become the standard communication and computing device used by large populations of the world. These smart phones are used to reach each other but also other devices and sources of information via a great diversity of new services provided in the Internet cloud, although the real-time voice communication call between people still is a very important and appreciated service.

Due to the network's great importance, the requirements on the components that constitute the system are several. Since so many users and much in our society rely on its services, the central parts of the network must always be available. The most important type of such a central component in the telecommunication network has for long been the telecommunication exchange. The exchange enables many calls to be set up and alternative paths to be taken if a preferred path in the network has failed, and hence should be very reliable. This reliability is probably the most important requirement of the exchange. The exchange should also be able to handle all signaling standards in a network to coexist with all equipment in the network, irrespective of age or fabrication. This imposes great requirements on compatibility.

Note that a telecommunication exchange can be a local exchange or a transit exchange, it is often placed in a central office, therefore sometimes called a central office, in other cases it referred to as a switch, the core part of the exchange. There are both public exchanges and private branch exchanges. An exchange is a node in a telecommunications network that includes functions for access, control, switching, and charging of calls and other similar communication sessions. These different parts may be physically separated and distributed, also to places outside the central office site; and act as subnodes connected via signaling protocols, for example, between access, control, and switch resources. A call as network connection setup concept is not just about phone calls but is a general connectivity and communication session service for two or more parties needing access to communication bandwidth between two or more points in the network over a shorter or longer period of time. This has evolved into convergence between the circuit-switched telecommunication networks and the international packet oriented data networks commonly called the Internet. Connection services can be established not only between peer users but also from users (human and machines) to central computing and storage servers available via the Internet. The Internet including the servers accessible within it is often visualized and referred to as the cloud.

A telecommunications exchange is a complex system. State-of-the-art hardware and software technology is used to create very high capacity exchanges in terms of the number of connected subscribers and number of switched calls. To evolve and maintain their services and service quality, several millions of hours of hardware and software development are invested each year. An exchange can connect hundreds of thousands of subscribers and switch a million of calls at the busiest hour during the day; and all of this is in real time, meaning short setup time and low delay of the voice. The quality of service requirements is thus great. Whether or not the call is made between two different continents, the call has to be switched through the network with low delays and provide good speech quality.

\section{HOW IT STARTED}

The basic service associated with traditional telephony is the bidirectional voice communication dialog between two persons located at different but fixed places. To enable such voice dialogs, each person needs a telephone set equipped with a transmitting device starting with a microphone that translates acoustic energy to electric energy and ending with a receiving device equipped with a loudspeaker that does the reverse transformation.

Each person who has a phone set also wants to be able to select whom to connect and talk with. For the establishment of such a connection service, one must be able to signal from each place to any other selected place, to tell that a connection is wanted between one calling and another called person or device. The traditional fixed telephone call is hence directed to a place with a telephone set (also referred to as telephone or phone) close to where the called person is expected to be located. To enable the sending and reception of alert signals the telephone is equipped with a tool that can generate an electrically encoded alert signal and a tool for reception and transformation of the electrical signal to an acoustic signal. For a basic two party dialog, two telephones are connected, electrically via a pair of wires. In the very beginning, the second wire was implemented by an earth connection; this, however, resulted in a high level of crosstalk, especially when several unshielded single wires came close to each other.

To start with, telephones were mainly used between just a few locations as between a shop owner's office, workshop, and home. A simple n-way selector switch could then be used by the caller to select another location within such a small local mesh structured private net (Figure 1). 


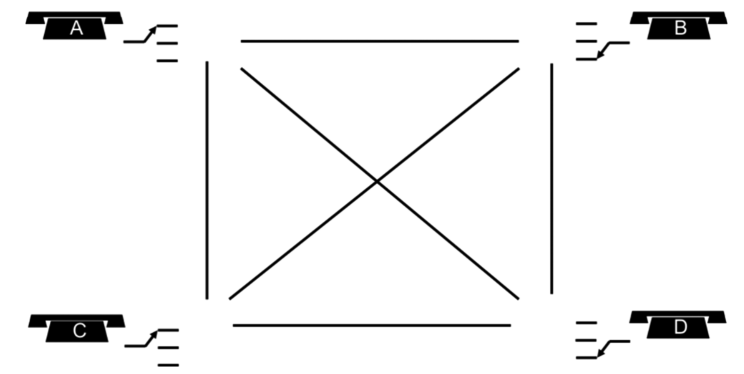

Figure 1. Local network with distributed three-way line selectors.

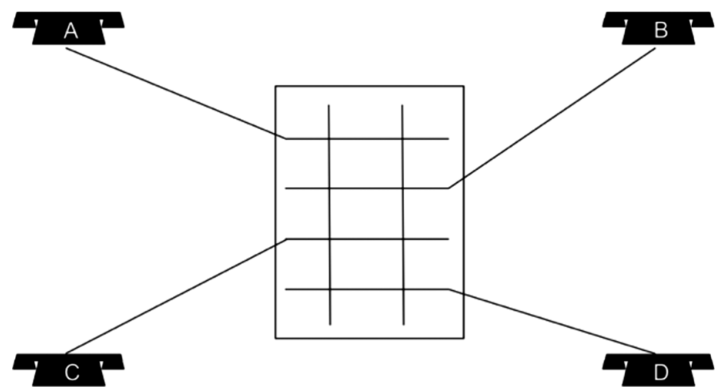

Figure 2. Connections between wires handled at a local exchange or switchboard.

Later when several families and organizations in a town had such connections, the communication possibilities were extended. This was enabled by connecting all telephones via their wires in a star structure to a central office equipped with an exchange consisting of a manual switchboard and a human operator (Figure 2). Decreased costs of cabling could be achieved by sharing of groups of wires going in the same direction, connecting the switchboards, called trunk lines.

Hence, each telephone in the local area was connected via a single wire or a pair of wires to the switchboard. Such a single wire or pair of wires is called a subscriber (or access) line or loop. The switchboards themselves were interconnected via other lines, called trunks. To enable voice signals to be carried with less distortion and over longer distances, the trunk lines soon came to use four wires, one twisted pair in each direction. This allowed the use of inductor coils for impedance corrections and amplifiers that refreshed the strength of the signals when the trunk lines where long. However, since the signals where analog some signal quality degradation was unavoidable, especially over longer distances.

The rapid growth of the telephony network during the early days is partly explained by the fact that the network technology had large similarities with the technology already used for telegraphy. But perhaps most important, the telephone set replaced the telegraph operator needed to read and encode every message, using Morse code, and thus simplified the human-machine interface and also made dialogs more feasible and private.
To establish a call, the calling person had first to send a ring signal to alert an operator and then tell the operator whom to be connected to. If the call was local, the operator then had to send a ring signal to the person being called and ask this person for permission to set up a connection from the person calling. The operator could at this point make clear by whom the call was to be paid. A call could then be established by connecting the two pairs of wires to each other. To simplify the operator's work, the manual switchboard was designed to make it easy for the operator to supervise the lines that were busy and to handle: the request for, the establishment, and the ending of a call. If the call was nonlocal, the operator first searched for a free trunk in the right direction and then alerted and called one of the operators at the receiving end of the trunk (using it as a signaling trunk) and asked for help to establish the call. Such searching, routing and forwarding of call setup tasks presume that the operator had knowledge about the networks topology. For a long-distance call a chain of operators had to be involved via a chain of point-to-point trunk lines (links) before the called person could be reached and asked for permission to set up the call via voice carrying trunks along the same path.

To set up a long-distance call, a number of resources must be free, including operators, signaling trunks, voice trunks, and switch board connection points. If some resource was lacking, the originating operator could serve the customer by organizing a waiting list or job queue and then set up, first in queue, calls when resources eventually became free. The operators had to keep track of each job by help of a written job record, including time stamps for charging purposes and also provide time supervision of the ongoing calls to guarantee that resources allocated to a call eventually were released even if the calling parties forgot to send an endof-call alert signal.

When the number of subscribers increased, quite a large number of human operators could work in the same exchange office. Different techniques were then used to ensure that they could share the workload and coordinate the setup of calls between subscribers connected to different switchboards. Parallel processing of call attempts could be achieved, for example, by distributing the incoming access attempt from a subscriber to a nonbusy switchboard operator. Another example is that one single operator could handle the setup of a local call within a large exchange consisting of many switchboards by use of multiple point technique - that is, by having the switchboard's all outgoing lines connected to all other switchboards in the office.

When the demand for telephony increased, the number of operators swelled. Pressure grew to decrease the costs for human switchboard operators but also the time to set up calls. Unsupervised operators could listen to and prioritize calls at will and thus requirements on personal integrity and fairness were also reasons to try to automate the call setup procedure. The first automated switches were based on the use of electromagnetic coils, effectuating drive mechanisms and contact points. These electromagnetic devices were, in their turn, controlled by signals generated from the telephone by use of a dial. The dial could generate 
sequences of current pulses, where the number of pulses corresponded to a dialed decimal digit, called decadic dialing. A subscriber was assigned a telephone number related to a corresponding access line number. The digits used to represent this number controlled the behavior of the switch. The signals were first decoded and used to control the switch movements directly using decadic dialing and somewhat later indirectly via registers. The use of registered signals reduced the requirements on timing of the signals, mechanical precision, and preventive maintenance, and also increased the flexibility by making number translations possible.

The digits where used to tell the address to a phone set in a network area, meaning that all decisions were not directly controlled by the digits. For example, a number of trunk lines between two exchanges could be treated as a group and the digits used merely to select and seize a trunk group going in the right direction, for example, to the right network. Then one could search (or hunt) for a free trunk line and, when available, select one free within this group. When no trunk line was free, an alternative group or route could be tried. However, the number of alternative routes was limited both due to economic reasons and because the networks soon came to be built more or less hierarchically to simplify the coordination work needed to establish nonlocal calls. If no free route could be found, a blocking situation occurred and the call attempt had to wait for resources to become free. The operator could then use different methods to supervise resource release events and to handle the queue of waiting call attempts.

It is interesting to note that the tasks performed by a human operator, such as searching for, seizing, monitoring and handling of resources, and charging records, were very similar to what the control system of a modern exchange does. There are also similar analogies between the call routing, redirection, and answering tasks made by a human operator and tasks performed by the control software in a modern stored program controlled automated exchange.

The network has evolved from very simple bidirectional communication links via small private mesh and star networks that as soon as signal regeneration and amplification technology permitted were interconnected via transit networks to larger more public, global, and hierarchical network structures. To enable several simultaneous calls between exchanges, one started to use trunks, to allow a simple kind of sharing called space division. To increase the capacity and limit the number of individual physical lines in a trunk, one had later to use other more advanced multiplexing techniques allowing several calls to share the same physical line. This evolution started with frequency division and has evolved via digital time division toward many different combinations of space, frequency, phase, amplitude, time, and code division (encoding). Sharing by help of multiplexing enables the creation of a logical network layer on top of the physical transmission media implementing a number of logical lines (or channels) and hence a more efficient use of each physical line.

Logically, signaling has always been separated from the voice connection. A trend has been to clarify this by a separation into a signaling network and a voice network. However, the signaling network may in practice use reserved logical lines or channels multiplexed on top of the same physical lines as the voice network.

Transmission and switching of the voice, has evolved from analog; via simple digital voice sample encodings to much more compressed digital encodings. The signal encoding has in a similar way evolved from the use of simple current pulses, via frequency encoded signals to digital message records.

Manual exchanges handled by human operators were quite flexible and intelligent in many ways since the primitive alert signals simply could be complemented by verbal communication between subscriber and operator - that is, human to human. Automation required a predefined signaling scheme, including not only alert signals but also encoding of the phone number of the called line. Structured network and numbering plans evolved. To make the automated exchanges able to provide enhanced flexibility and more advanced services, the first decadic control of an exchange directly from signals representing digits evolved via register mapped control to stored program control of the exchange behavior. The automation possibilities were increased further by introduction of larger signal alphabets and protocols capable of much more than just alert signals and digits directly corresponding to line numbers.

A great step from a functional point of view was the introduction of radio transmission enabling wireless access to/from mobile terminals. This allowed a call to be directed to or from a mobile terminal carried by a person also when on the move rather than just to or from a fixed phone set and place (terminating a wire or fiber).

Dividing the geographical area to be covered into regions called cells, another example of space division, enabled a great capacity improvement by allowing radio frequencies to be reused in nonadjacent cells. Technically it was not new to reuse the radio spectrum by dividing an area into cells, but connecting the cells covered by base stations to the telecommunication network was innovative and created many new challenges and opportunities. Other steps in this direction are digital subscriber lines; new call services (e.g., for redirection of calls) sometimes substituting what a manual operator previously could give help with; personal numbers that in conjunction with mobility services make it easier to reach a specific person rather than a phone terminal and also can increase the competition among operators if the phone number becomes a property of a person rather than an operator; the merger of telecommunication and data communication networks enabling new multimedia communication services.

A recent such network convergence trend, for example supporting the evolution of streamed and real-time multimedia services, is the sharing of a more and more common infrastructure for fixed and mobile voice, data, and audio and video services. In this new setting, some of the functions of a telecommunication exchange become obsolete, or replaced by virtualized counterparts, and other more dedicated nodes become more important. For example, circuit switch functions are replaced by 
packet forwarding, and new forms of access control, authorization, authentication, address location, and charging services are introduced. This development along with international standardization is also believed to increase the competition between different equipment suppliers.

An exchange is typically a quite general node that can include almost all functions needed in a telephone network. However, one can also distinguish specialized network nodes. Early examples of these specialized nodes are local exchanges and transit exchanges. Today, one can also distinguish other types of nodes such as network access nodes; switch, call, and service control nodes; mobile switching nodes; and network database nodes (e.g., databases for number translations; handling of subscriber service profiles; location information supporting mobility of subscribers, authentication data, authorization data, charging data, and equipment data). Other network nodes and functions are different types of information service, media content, e-trade transaction, and access right handling servers.

\section{THE EXCHANGE FROM A NETWORK PERSPECTIVE}

The main purpose of a telecom network is to interconnect users via their phones, but also computers, devices, and services. Since most users does not use the network all of the time, many network resources can be shared among the users.

A large exchange is a means to share switching resources by keeping many shared resources in one common pool, thus providing statistical gains. A large exchange or pool of resources will reduce network costs by enabling the sharing of network resources and reducing the number of lines, transmission capacity, and external signaling needed. This compared to if split into smaller exchange nodes and other specialized nodes.

The concept of an exchange can be related to the functions of a network node where many telecommunication lines are connected. However, the traditional functions associated with an exchange can be distributed and located also to other node types in the network. This is a trend that today seems to evolve further based on concepts such as network virtualization and network function virtualization (NFV) where software-defined virtual networks and virtualized computers, executing on top of shared physical network and computing resources, provide virtualized functions to the end users. More about this in the section called Evolution Trends.

\subsection{Functional, Structural, and Physical Perspectives on Networks}

A practical approach is to look at networks from the operator's perspective. From this point of view, a telecommunications network can be divided into four major networks: an access network, a transport or core network, a signaling and control network, and a management network. In more recently developed networks, one often complements this view with service and media networks.
However, for different purposes and reasons, one may also talk about other types of networks or views that are related to each other in different ways. A reason to do this is that one often needs to look at networks from several perspectives such as functional, structural, and physical.

In abstract functional perspectives, one views the network from the external environment as if the whole network were a black box or a cloud and focuses mainly on the services that the network provides. Functionally, a network can be defined as a set of points at which functions with certain properties are accessible. A network function provided at one level may be implemented by using one or more functions at the adjacent lower and more concrete network layer (Figure 3).

In structural perspectives, one takes a closer, more internal, detailed, and structural (white box) view and focuses on matters related to structurally measurable properties, including how the network functions are partitioned, logically distributed in space, and allocated to network nodes. Structurally, one can hence define a network as a set of network nodes, each containing a set of node functions, connected via node internal connections to implement composed functions where some are made externally accessible via external connection points. The internal connectivity enables the node functions to be composed and to cooperate so as to implement the network functions provided externally.

From a physical perspective, the actual links and nodes (implementing logical/virtual functions) and their concrete placement in the physical world are made clear. Properties related to distances, areas, and capacities of different sections of the network can be described.

The functions available at the network points can for economical or other reasons be colocated in the same physical node or site. The networks are related to and use each other in many different ways. For example, the signaling network is used to enable functional interwork between the other networks; a subscriber can, by sending signals via the access network, obtain access to functions provided by the service network that establishes these services using (by sending of signals to) the connection network(s); the transport network is used by all the other networks as a bearer for transport of in different ways encoded data of all kinds.

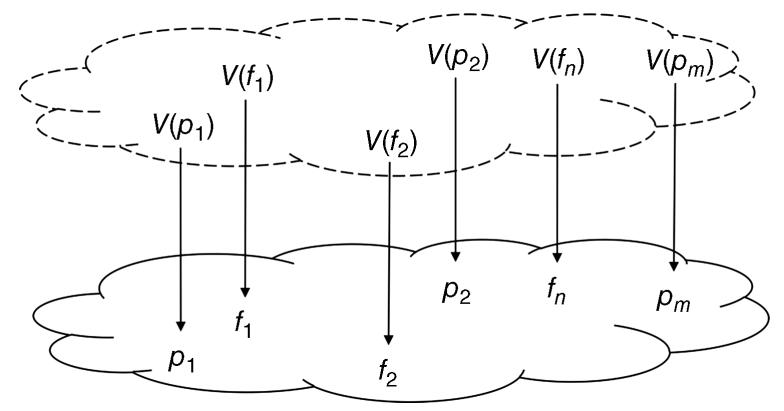

Figure 3. Network function access points and related functions in higher (more abstract or virtual) and lower (more concrete or physical) network layers. 


\subsection{Network Design}

As discussed above, there are several types of networks seen from a functional point of view. A purpose of this partitioning into several functional networks is to help to build and maintain a network architecture with welldefined functional areas and interfaces between these that simplify extensions and modifications of the network and its functions. Another purpose is to support the building of cost-effective networks, both from a purchase and from a life-cycle point of view, enabling low operation and maintenance costs.

The end users of a telephone network have a more holistic view. They want to be able to contact each other so that they can talk or exchange data when they want even if physically far apart. Hence, the main purpose of a telephone network has been to enable real-time voice communication between people. In a mobile multimedia network, this extends to having mobile access also via other media and to other services wherever the user is located when on the move. This includes access to servers such as ordinary databases and file systems as well as streamed audio and video content servers part of the Internet. This now extends to machine-to-machine (M2M) communication and Internet of Things (IoT) gateways that enables many things that we use in our daily life including our cars and home appliances can be wirelessly connected to and be made accessible via the Internet and related services in the so called cloud.

End users of this integrated network not only want to talk; they also want to talk and exchange data inexpensively and with a good quality of service, including privacy and security. This requires efficient use of resources and a suitable network design. The costs to operate a network depend much on how the subscribers are distributed in the geographical area that the network is aimed to cover. Charging policy, traffic intensity, and traffic patterns, such as length and locality of calls, are other factors that all influence the design of the network. Most networks are built in a more or less hierarchical structure to collect, concentrate, transmit, and connect the traffic such that higher capacity transmission and switching equipment can be utilized. This reduces the costs for transmission and switching, since many users can then use the equipment more efficiently and with less risk for blocking due to statistical multiplexing gains. The degree of concentration that can be utilized is influenced by the traffic intensity and the desired service quality level.

Another important cost factor is that the total physical length of the transmission path can be reduced in this way, and this is important since the cost for digging ditches and make use of pipes to host cable and fiber links is a large contributor to the overall network costs. In some areas, microwave radio links are used to reduce cost where it is too expensive to dig ditches. For high traffic routes, direct routes are often used, especially if the distances are short, such as in metropolitan areas. Alternative routes are also used to improve reliability, but these increase the cost and for that reason are usually not afforded in the access network. Using the structural perspective of networks, one can for a specific area identify a distribution of subscribers that must be interconnected in a reasonably efficient way - for example, in a star or ring-structured physical access network. One can then allocate a number of network nodes of different basic types that are needed, both to deal with expected maximum traffic loads and to interconnect these in a way that keeps transmission costs close to a minimum. The structure of a modern fixed and mobile telecommunication and data network is illustrated in Figure 4.

We now go back to the external functional network perspective and especially to how the different functions are distributed in the physical network. This distribution can be guided by different principles. Simple and cheap functions that are used often and for long periods of time should be located close to the subscribers. Complex and expensive functions used seldom and for short periods of time should be placed more centrally in the network and thus be shared by many users. However, with today's technology and using mainstream components, complex must not necessarily mean expensive and hence a complex function if implemented as an integrated circuit can often be placed close to the subscribers, for example, voice coders. Furthermore, the effects of distribution on reliability, signaling, and maintenance must also be considered when deciding on function distribution over network nodes.

From the above discussion, one can see that there is no absolute definition of how networks shall be implemented or what a network node shall contain. Rather, there are several possible configurations that can fulfill the requirements, because not only does the distribution of subscribers as well as their quality of service (QoS) requirements and traffic patterns differ significantly but also implementation costs for different types of solutions differ and change over time.

Going back to and analyzing what an exchange is, one can see that many of the functions carried out in the different logical networks have traditionally been placed in an exchange node and can still be located in such a general node. On the other hand, these functions and hence the exchange can also be distributed and placed in more specialized networks and nodes. With well-defined functional areas and interfaces between these, it is possible to configure networks and network nodes in many different ways. However, for a node or cluster of nodes to be regarded as a telecommunication exchange, it is assumed to have some basic call access, control, switching, connection handling, and charging capabilities.

\subsection{Routing and Switching}

The task of finding a path (or route) from source to destination is called routing. The task to follow a path from source to destination guided by end-to-end address information is called path selection. Each node that participates in this simpler forwarding task does not need to know the whole path but must be able to analyze the destination address or a path identity find out to which link or outgoing port the switching or forwarding should be made and also find such a link with a trunk that is free to use. 


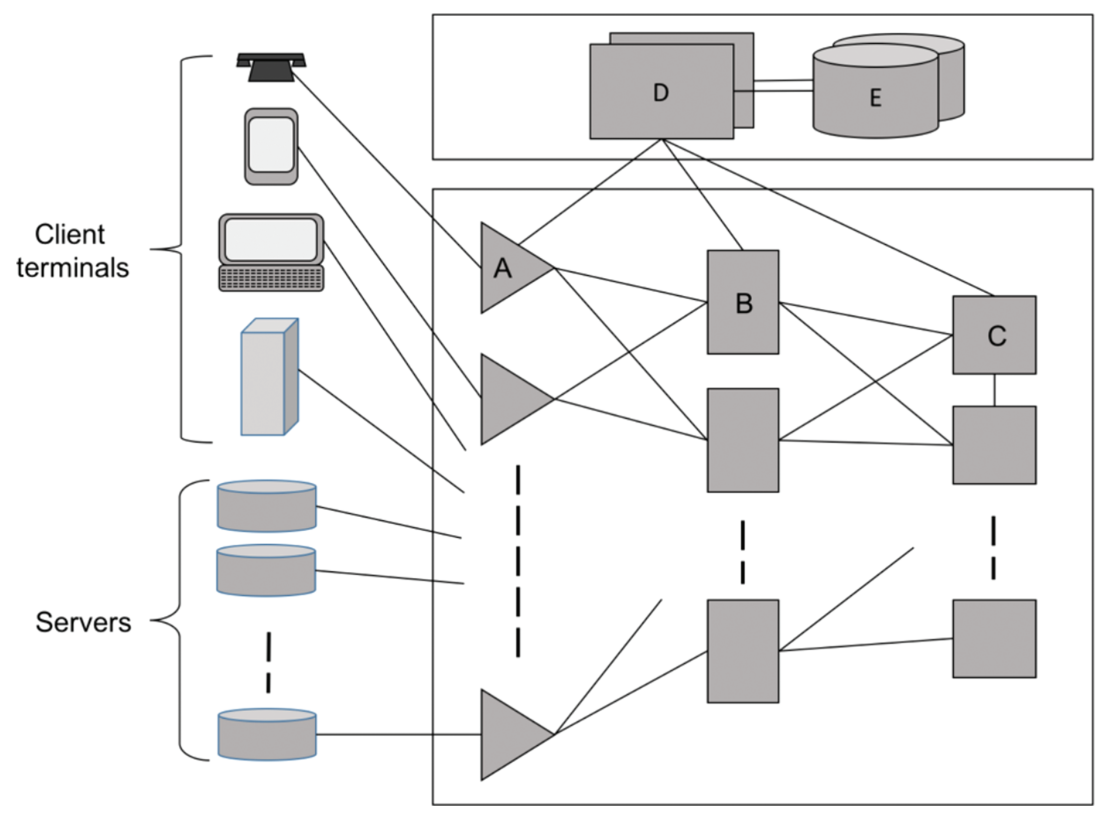

Figure 4. Integrated telecom and data network, where nodes type A are base stations or subscriber line access nodes; B are local exchanges, mobile switching centers, and routers/switches; C are transit exchanges, media gateways, and core routers/switches; D are network control centers and $\mathrm{E}$ are network topology, phone number, network address, subscriber data, and locations registers.

To forward information (a bit, a voice sample, a message, a cell, a packet, or a frame) from a fixed or mobile input terminal (address, channel, line, or trunk) to a selected output terminal (address, channel, line, or trunk) of an exchange where the path selection or forwarding is controlled by address (or path) information (helping to follow a selected path or circuit) is called switching.

The telephone exchange will use network topology or routing information to find and select a path and prepare a connection. Hence, routing precedes the establishment of a full circuit-switched connection and is done only once for each call setup, while switching is done for each voice sample or packet when using the connection for transmission of voice or data information.

To guarantee that all resources needed to enable communication in real time are available when needed, they must first be reserved or seized. This is by setting up a circuit connection and by finding the free resources needed along a route between the end points of the connection. Information to be exchanged can then be sent along the circuit connection. The connection is thus first routed and then set up before the actual information exchange starts and then it is released when the information exchange ends. This guarantees that all resources needed to enable voice or data communication in real time are available and reserved before the actual transmission can start.

For historical reasons, circuit connection techniques are often associated with the synchronous transfer mode (STM) and pulse code modulation (PCM) techniques using a $125 \mu \mathrm{s}$ frame rate for transmission of byte (8-bit) encoded voice samples, recorded at $8 \mathrm{kHz}$ sampling rate.
Another technique, now part of the history, called asynchronous transfer mode (ATM), used small packets called cells with a given fixed size (53 bytes) divided into a small header ( 5 bytes) and payload (48 bytes). The header contains addressing information based on path and channel identifiers, where a path is a bundle of channels. Routing is done in a setup mode where the destination address is sent as payload in a control cell (hence used to carry control information). The traffic carried in user data cells can then be switched at the path or channel level in each node along the paths and channels routed and set up in advance from sources to destinations.

A third now dominating technique used for data communication is to send information in packets of a limited size, for example, as Internet Protocol (IP) packets. Using this technique, one can attach addressing information and other control information into a packet header and a body of user data and send it via a route going toward its destination address. Each node on the way to the destination participates in the forwarding of the packet in the appropriate direction. For larger pieces of information that needs to be divided into several packets, forwarding is done for each packet in each node. To get more deterministic packet transport delays on top of IP networks virtual, circuit like, reserved routes can be established using multiprotocol label switching (MPLS) techniques and protocols.

\subsection{Network Standards Evolution}

The classic telecommunications network available all over the world has been called the public switched telephone network (PSTN). It is basically designed to enable setup of 
calls and transmission of speech between two or more users. However, this network is also used for facsimile and data traffic (sent via modems over the analog parts of the network). Examples of call services are alarm calls and abbreviated dialing, call forwarding, and three-party calls.

Integrated services digital network (ISDN) was an evolution of PSTN that gave the subscribers digital access to integrated or combined services. ISDN aimed to integrate different telecommunication services into the same network that transports voice and data in digital form between network access points. The evolution from analog to digital end-to-end communication gave safer and more flexible transfer of information. ISDN provided a range of services divided into bearer services and teleservices. ISDN is based on the digital telephony network using ordinary two-wire subscriber lines, 24 or 32 channel PCM link structures, and Signaling System No. 7. Integrated access gives the user access to both voice and data services through a single subscriber line, whereas combined access allows the use of several subscriber lines. Services include voice, facsimile, and computer connections. ITU-T defines two types of usernetwork accesses: Basic Rate Access, used for low traffic load, including one $16 \mathrm{kbps}$ signaling channel (D) and two 64 kbps communication channels (B); Primary Rate Access, supporting higher traffic loads, normally including 23 (T1) or 30 (E1) $64 \mathrm{kbs}$ communication channels (B) and one signaling channel (D).

Today ISDN, at least as a service that the end user is aware of, to a large extent is replaced by different Internet and IP protocol-based services with much higher bandwidth although the ISDN services and protocols still sometimes are used to implement links in and access to what we call the Internet.

The public land mobile network (PLMN) is used as an acronym for all cellular mobile networks with the primary objective to provide wireless communication to and from mobile subscribers connected to the fixed network via radio. The radio interface is implemented by the mobile terminals and the base stations implementing a wireless access network. The base stations are end points in the fixed (wired) network.

Early analog mobile networks based on standards, such as advanced mobile phone system (AMPS) and the nordic mobile telephony (NMT) are now called the first generation (1G) and their digital versions (DAMPS); global system for mobile communication (GSM) and personal digital cellular (PDC) are referred to as second-generation (2G) cellular mobile networks. The third-generation (3G) systems have increased the bandwidth and capacity based on code division multiple access (CDMA) and wideband code division multiple access (WCDMA), such as IMT-2000 and UMTS are designed to support variable speed multimedia communication. Today, we have the fourth-generation (4G) systems that have been developed and deployed step by step following the ETSI long-term evolution (LTE) roll out plan also managed by the 3GPP (3rd Generation Partnership Project). 4G uses more advanced coding and modulation schemas and has also got some additional frequency spectrums to use, all resulting in higher bandwidth and capacity.
Important types of network nodes in the PLMN are the following:

- Mobile services switching center (MSC) - controlling the calls within the PLMN as well as calls to and from other tele- and data-communication networks.

- Home location register (HLR) - database handling information about which supplementary services a subscriber has activated and in which MSC area the subscribers phone is currently located.

- Visitor location register (VLR) - database maintaining information about the locations of the active phones in the area controlled by the MSC. The VLR fetches information from the HLR so that the call setup can be performed without using the HLR each time.

- Media gateway (MGW) - switch, router, and translator (of coded media) facilitating communication between RNCs, the core network nodes, and O\&M nodes.

- Base station controller (BSC), called radio network controller (RNC) in UMTS, coordinates and controls a number of radio resources usually located in base stations and some interwork functions such as handover between the cells, covered by the base stations.

- Radio base station (RBS) - radio transceiver enabling communication with the active MS in the cell.

- Mobile station (MS) - that is, the mobile phone.

Based on the separation of the network in a control signaling layer and a user data transport layer, the intelligent network (IN) is an architecture aimed at making the telecom network to work as one integrated system where new network services can be developed, introduced, and made available from a central service control point (SCP). The IN architecture aims at a logical separation of signaling, call, connection, and transport. The idea is that local and transit exchanges do the initial number analysis and then ask the SCP to handle the call, unless it is a simple IN service that can be handled locally. The SCP then executes a service script that results in orders to the exchanges along the connection path telling how to proceed with the call. For this communication, the IN application protocol (INAP), that is a part of the signaling system number 7 (SS7) protocol stack, is used. Services are defined by service scripts or building blocks, including functional components developed by the operator using a service design and development environment.

The telecommunication management network (TMN) is an architecture and standard portfolio aimed to support operation and maintenance. It defines functional areas and protocols for management in general terms and also more specific information models for how to monitor and manage these functional areas.

The open systems interconnection (OSI) reference model is a standardized layered model of how computer systems can be interconnected and interoperate that has had and continuous to have a strong influence on the way signaling networks and their protocols are built. The model defines seven protocol layers: (7) application, (6) presentation, (5) session, (4) transport, (3) network, (2) link, and (1) physical. 
The telecommunication information networking architecture (TINA) is a consortium defining an open architecture for telecommunication systems. It focuses on the software architecture. To some extent, this effort can be seen as an attempt to put together some other standardization efforts such as OSI, IN, and TMN from a software architecture point of view.

\section{THE FUNCTIONS OF AN EXCHANGE}

The telecommunications exchange has evolved into a multiapplication digital node that offers its services to connected subscribers but also to the operator of the exchange. One example of a service offered to the subscribers is telephony calls, and a service offered to the operator is the ability to charge for services by registering of charging data in the exchange.

A telecommunications network offers various services to the subscribers and the network operator. ITU-T has divided these services into two main categories:

- Bearer services for transport of speech and data in the network between the user interfaces. The transport of speech is to be done in real time with negligible distortion or alteration of the speech. The bearer service supports the OSI levels 1-3.

- Teleservices that combines the bearer service with terminal services, such as information processing functions. Some teleservices are tied to a specific bearer service, whereas others utilize different bearer services. Examples of basic teleservices are telephony, facsimile, and computer connection.

The bearer services and the teleservices are divided into basic and supplementary services. Telephony is an example of a basic teleservice, and call waiting is an example of a supplementary service that gives users additional functionality.

\subsection{Basic Telephony}

Below is a brief description of the main functions required by the exchange in order to set up, maintain, and disconnect a basic telephone call between two mobile or fixed subscriber terminals.

Signaling. Early signaling was made in-band, on the same line where the speech was transmitted. This first by decadic pulses and later coded as tones of different frequencies. A still common in-band signaling system is multifrequency signaling, where a combination of two tones is sent to a tone-receiver in the exchange. Modern signaling is based on digital message passing. The globally dominant signaling system for telephony is the Signaling System No. 7.

Subscriber Signaling. To request the set up a call, the calling subscriber alerts the exchange and then sends the dialed number. For an analog access, the alert is made by the user lifting the handset, when the exchange detects this it replies by returning a dial tone. The dialed digits are then sent one by one. For a digital (mobile, ISDN, or VOIP) access, the alert and the digits are all sent in one message, this saves bandwidth and decreases the delay for call setup. In both cases the exchange replies with a tone to the calling subscriber when the status of the called subscriber has been checked.

Number Analysis. The A-number (the calling subscriber) and the B-number (the called subscriber) are analyzed and used as input to routing and charging analysis.

Subscriber Category and Service Analysis. The exchange will early in the call setup check whether the calling subscriber has any call service invoked, such as blocking of outgoing calls. The services are sometimes executed in the local exchange used by the calling or called subscribers, in other cases in a transit exchange or in a separate exchange that handles intelligent network (IN) services such as in a service control point (SCP).

Charging. Charging can be divided into two steps: analysis and output. The analysis is influenced by the charging method (detailed billing, toll ticketing, pulse metering, or flat rate) and the charging rate, depending on a number of calls related data set by the operator. The output includes formatting of the charging data along with transfer to a reliable storage medium, locally or in a charging and maintenance center. With exception for advanced services, there has been a trend toward simpler more flat-rate-based charging principles.

Routing Analysis. Finding a path from source to destination is called routing and is made mainly by analysis of the B-number. Usually, there are two or three (and sometimes up to ten) alternative routes to select among. The selection of a route (trunk group) is guided by priority and load status information. If the first route is already reserved, the next alternative is selected. There are sophisticated routing algorithms that dynamically (by checking the load) choose a link in order to minimize the congestion in the network. These dynamic routing algorithms can be either local or central; the local provides results by using data available in its own exchange such as previous success rates on different link choices, while the central algorithms collect input data from other exchanges in the network.

Connection. Traditionally, this meant the through connection of two $64 \mathrm{~kb} / \mathrm{s}$ circuits, one in each direction, established by the switch. Today, the bandwidths can be much larger to support for example real-time video sessions. A connection is required to be with limited probability of blocking, from end to end. This means that the switch fabric must add very low blocking probabilities, in order to fulfill the end-to-end requirements for calls that pass several exchanges. The connection also must be well synchronized with the exchange and with the rest of the network, in order to handle real-time digital speech and video connections properly. 
Trunk Signaling. Trunk signaling enables a call to be connected between subscribers connected to separate exchanges. This by sending signals such as alert messages telling that a call is to be connected or disconnected along with routing information, relevant parts of the dialed digits. Modern signaling systems also support detailed billing, advanced network services, and transparent user data.

\subsection{Subscriber Services}

A modern computer-controlled exchange that communicates via digital messages enable almost any service to be evolved. However, the service software is not only located in the exchanges but more and more also in terminals, service control points, and network databases. The most cost-efficient location depends on the type of service. For some services where the logic is local (such as abbreviated number, also called speed dialing), it is efficient to do the translation between abbreviated number and real number directly in the calling terminal. For more advanced network services such as virtual private networks, freephones, or universal personal numbers, it is more natural to locate the service logic in a network node, in either ordinary exchanges such as the mobile switching center or fixed local exchange or in other cases in a network database such as the SCP or HLR. The advantage of central service control is that the introduction of new services and features is simplified. In addition, some features require consistent data for the entire network, such as the information in the HLR regarding where a called mobile subscriber is located.

More powerful protocols enable more advanced services to be implemented in the network. At the same time, there are an increasing number of services that now are implemented in the terminals, and related data is sent transparently through the network between the end users. As an example, ISDN did not took off while transport of data traffic over the telecom network has increased much more rapidly, where the services are executed in the end-users' computers or smart phones.

Examples of common telephony subscriber services are Freephone, the call is free of charge and instead paid by the called party. Conference Call: more than two parties can take part in a call. Transfer Services: the call is transferred to another telephone immediately or when the called number is busy or not replying. Universal Personal Number: one phone number is used regardless of which mobile terminal or fixed physical connection a person is using. Call Completion Service: when the called party is no longer busy or nonreplying, the call is reinitiated. Virtual Private Network: a group of subscribers, for instance a corporation, form a private network with their own charging and telephone numbers.

\subsection{Cellular Mobile Telephony}

Cellular mobile telephony differs from basic telephony, since the subscribers can move freely within the areas covered by the radio access network. As a result, the exchanges in a cellular system must keep track of where the subscribers are located and find free radio channels to use for setup of new calls and during calls when the subscribers are moving between cells in the covered areas.

The radio frequency spectrum available for mobile telephony is a scarce resource that is reused by dividing the space in small areas called cells. The frequency spectrum is divided into frequency bands, and these bands can in turn be time or code divided into channels. Due to the use of limited power levels, not adjacent cells can reuse frequency bands and channels without disturbing each other.

Handover. Handover means to change or switch cell from one cell to another with better radio transmission quality during an ongoing call. The handover decision is based on measurements of received signal quality in up and down links. Handover can be made several times during a call. This and the fact that handover decisions require the collection and analysis of measurement data contribute to making cellular mobile telephony more processing resource consuming compared to fixed telephony.

Channel Allocation. Channel allocation, aims at finding and assigning free frequency, time and/or code divided channels within a cell to calls. The channel allocation logic gives an ongoing call higher priority than a new call. Channel allocation is closely related to the handover function and especially intercell handover, which does handover between allocated channels in the same cell.

Location Update. When the mobile phone is turned on and running in idle mode, it listens to control messages indicating which location area the closest base station sending control messages belongs to. When a location area border is passed, it sends a message to the mobile switching center (MSC), indicating that the subscriber has moved to another location. The information is stored by the MSC, in a visitor location register (VLR), and it is also stored in the home location register (HLR) if the area is handled by a new MSC.

Paging. Locating a mobile subscriber within the network is called paging. This is done by requesting the HLR in which VLR/MSC and location area where the subscriber is located, and then sending a paging message on a control channel called the page channel to all the cells in that area.

Roaming. When subscribers move to another operator's network than their own, the network can page the subscriber and then set up a call to their new location.

\subsection{Operation and Maintenance}

A modern telecommunications exchange is supported by operation and maintenance functions that guarantee a high quality of service to the operators and the subscribers. Operation is the normal everyday running of the exchange. This includes activities to adapt the exchange to continuously changing demands. Examples of operational activities are connection and disconnection of subscribers; change of subscriber data; collection of charging data; and collection of statistics. 
Maintenance includes the prevention, detection, localization, and correction of faults. The faults can be detected automatically by the exchange or reported to the operator by the subscribers or other exchanges in the network. Examples of maintenance activities are fault detection, testing, and repair of exchange hardware, for example, trunk lines or subscriber lines; fault detection, auditing, recovery, and correction of exchange software and data; checking of disturbance indicators in various parts of the exchange, such as the power supply system or the control system.

In a modern telecommunications exchange, there are several approaches to maintenance. One is preventive maintenance that involves a set of routine tasks to check for faults before they occur; this requires a high level of effort to achieve a certain grade of service. Another is corrective maintenance where faults are dealt with as they occur; this requires a more selective and limited effort but may result in a less consistent quality of service. The best is to have a proper balance between the preventive and corrective maintenance.

Statistics are used to supervise traffic load and performance in the network and to reconfigure an exchange. This to change or tune its behavior, capacity, or quality of service. Particularly for the configuration of location areas and cells within a mobile network, large amounts of traffic data are used as evidence to support the right corrective actions.

All operation and maintenance activities should have minimal impact on the traffic handling of the exchange. The ideal situation is an exchange where every subscriber can make a call at any time no matter what happens to the system. In order to achieve this, the system should be robust to operator errors and allow the performance of maintenance and software and hardware upgrades without affecting the execution of traffic events.

An exchange should be able to be operated and maintained remotely. The ability to access exchanges remotely using an operation and support system (OSS) ensures a high level of service to subscribers and low costs for the operator. A centralized operation also contributes to a more reliable operation of the exchange and a reduction in personnel.

\section{ARCHITECTURE OF COMPUTER-CONTROLLED EXCHANGES}

The architecture of modern stored program computer-controlled exchanges is influenced to a large extent by the central control system and its relation to the decentralized regional control of the switch as well as signaling and transport trunk resources (Figure 5).

\subsection{Modularity}

A modular architecture is typically desired to lower the costs of system handling and make it easier to adapt the system to the changing world of telecommunications. In a truly modular system, each module is fully decoupled and

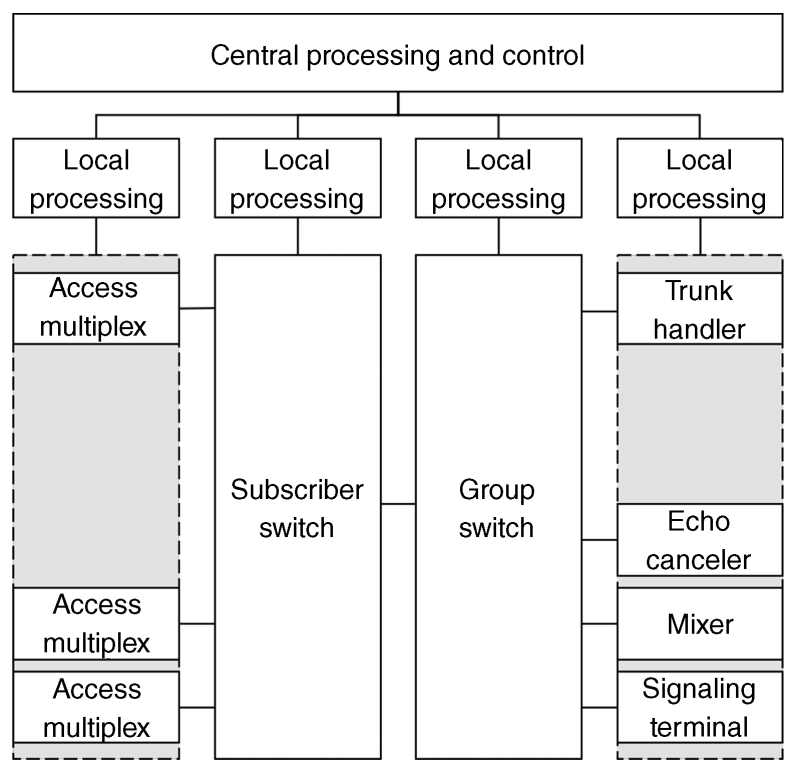

Figure 5. Typical architecture of stored-program-controlled exchange.

independent of the internal structure of other modules. There are different forms of modularity, for example:

Application modularity: To support the encapsulation and management of several larger applications in one node.

Functional modularity: The system is defined in terms of functions rather than implementation units. Functions are possible to add, delete, and change without disturbing the operation of the system.

Software modularity: The software modules are programmed independently of each other, and interact through defined interfaces and protocols. New or changed modules can be added without changing existing software.

Hardware modularity: Hardware can be added or changed without affecting other parts of the exchange and its operation.

Typically, the implementation of an exchange can be divided into Application modules, Resource modules, and Control modules.

\subsection{Application Modules}

On the highest level, the system architecture of the exchange can be divided into various application modules in analogy to how telecommunications nodes interact and communicate, using protocols enabling modules to be added or changed without affecting other modules.

The application modules implement various telecommunication applications and have standardized interfaces to resource modules. Application modules thus act as clients to resource modules. In general, an application consists of functions for access and service. Examples of application modules are analog access, digital access, mobile access, PSTN services, ISDN services, MSC 
services, and home location register (HLR). This list may be extended with newer functions such as IP-TV distribution services.

\subsection{Resource Modules}

To support the applications modules, the resource modules handle and coordinate the use of common resources available to applications by means of well-defined interfaces to the users. Resource modules act as servers giving control of resources to application modules and may contain both software and hardware. An important part is the group switch. Remote and central subscriber switches (RSS and CSS, respectively) as well as trunks are connected via the group switch. The trunks connect the switch to other switches, to data networks, to mobile base stations, and so on. The subscriber switch handles the subscriber calls and concentrates the traffic.

The group switch resource module includes functions for selection, connection, and disconnection of concentrated speech or signal paths. Overall control of the group switch is performed by the central processor system. Regional processors take care of simpler and more routine tasks, such as periodic scanning of the hardware, whereas the central control system handles the more complex functions. Associated functions included in the group switching resource module are network synchronization devices and devices to create multiparty calls.

The subscriber switch resource module handles selection and concentration of the subscriber lines; transmission and reception of speech and signaling data to and from the subscriber equipment (e.g., on-and off-hook detection); multiplexing and concentration of the subscriber lines to save hardware and make more efficient use of the communication links between the subscriber stage and the group switch. The subscriber switch should be modular and enable to combine different forms of access in the subscriber stage. The subscriber switch can be colocated with the group switch in the exchange (central subscriber switch, CSS) or located at a distance from the exchange (remote subscriber switch, RSS).

The remote subscriber multiplexer (RSM) is an add-on subscriber access node, used in the access network, to serve small groups of subscribers. It provides both mobile and standard telephony connections. The RSM multiplexes and concentrates the traffic to the subscriber switch but does not carry out traffic switching functions.

The trunk and signaling resource module includes circuits for connecting trunks and signaling devices to the group switch. It also handles the adaptation to different signaling systems, namely, common channel signaling as well as various register and line signaling systems.

The traffic control resource module contains the traffic handling and the traffic control functions of the exchange. This module is responsible for finding the most suitable route between calling and called subscribers and of verifying that call establishment is allowed.

The operation and maintenance resource module enables tasks such as supervision of traffic, testing of the transmission accessibility and quality, and diagnostics and fault localization of devices or trunks.
The common channel signaling resource module includes the signaling terminals and the message transfer part (MTP) functions for common channel signaling systems such as SS7.

The charging resource module is used in exchanges that act as charging points. Pulse metering and specified billing (toll ticketing) are often offered. It is often possible to charge both calls and services based on usage of subscriber services and supplementary services as well as activation/ deactivation of subscriber services and supplementary services.

\subsection{Control Modules}

Control modules provide operating system functions, input-output functions, basic call service functions, and so on. The primary function of a control module is to provide the real-time processing and execution environment required to execute software in application modules and resource modules used to perform traffic-handling functions and call services. The processing can be centralized where one processor takes care of all tasks, or distributed where the processing of information is distributed over several processors.

Execution of telecom software imposes stringent realtime requirements on the control system. Call attempts arrive stochastically, short response times are needed, and overload situations must be handled. The main control modules are the central processor(s); the data store to store call data; and the program store to store the actual programs.

In order to achieve an efficient overall control system, it can be divided into the following:

Central control: One or more processors that perform complex program control and data-handling tasks such as execution of subscriber services, collection of statistics and charging data, and updating of exchange data and the exchange configuration.

Regional control: Distributed processors that perform routine, simple, frequent, and repetitive tasks, for example, input/output processing and protocol handling. They are of different types optimized for their main tasks and often have strict real-time and throughput requirements.

\subsection{Switching Techniques}

Until about 1970, most switches were analog and based on electromechanical rotor switches and crossbar switches. Since then the digital techniques have become dominant. Digital switches have been based on synchronous time and space multiplexed circuit switch technology. For data communication, packet switching technology is mostly used. In order to allow voice and data to share a common infrastructure, asynchronous transfer mode (ATM) was developed but has now largely been replaced by IP technology. Synchronous circuit, packet and ATM cell, and IP packet traffic are often carried by synchronous transfer mode (STM) carrier and synchronization technology over longer 

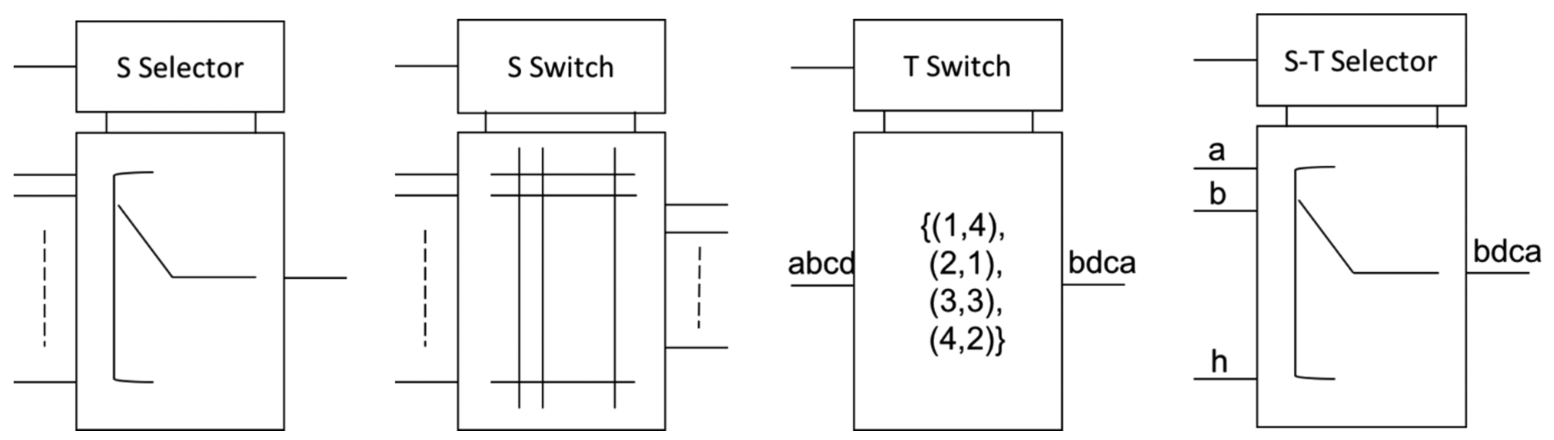

Figure 6. Examples of selection in space (a), switching in space (b), switching in time (c), and selection from space to time (d).

distances often complemented by Ethernet technology in the local access areas.

A space selector selects one out of several incoming lines (Figure 6a). A space switch connects physical lines in the space domain (see Figure 6b) and a time switch changes the ordering sequence of data (voice) samples in the time domain, illustrated in Figure 6c. A space to time selector time-multiplexes several incoming lines on one line (Figure 6d).

An STM-based switch architecture is made up of a combination of time and space switches ( $\mathrm{T}$ and $\mathrm{S}$, respectively). The switch elements $\mathrm{T}$ and $\mathrm{S}$ can be combined in several ways to realize a high capacity switch and make it configurable in many ways. Usually, time switching is used in input and output stages and space switching is often used in central parts of a switch. This basic time-spacetime (TST) switch structure can be used both in subscriber and in group switching stages. The first part of a TST switch is a time switch, which interchanges time slots between the external incoming digital paths and the space switch. The space switch connects the time switches at the input and the output. The last part of the TST switch is typically another time switch, which connects the time slots between the external outgoing digital paths and the space switch (Figure 7).

The time switch moves data contained in each time slot from an incoming bit stream to an outgoing bit stream but with a different time slot sequence. To accomplish this, the time slot data is temporary stored in a data store (DS) memory and then placed in a new time slot position. These

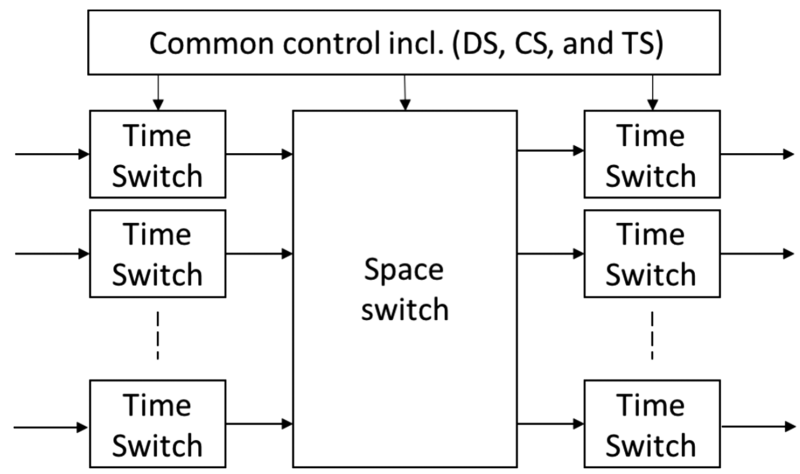

Figure 7. Typical STM switch with TST structure. operations need to be controlled by information stored in a control store (CS) memory. The timing of DS and CS is controlled by a time control (TC). Examples of control actions are time slot busy or time slot idle.

A typical space switch consists of a cross-point matrix that realizes the switching of time slots in space. The matrix can be divided into a number of inputs and a number of outputs and is synchronized with the time switching stages via a common clock and a control store.

Switch architectures based on asynchronous transfer mode (ATM) was designed to handle small packets, called cells, with a fixed size (53 bytes) divided into header ( 5 bytes) and payload (48 bytes). The header contains virtual path identifiers (VPIs) and virtual channel identifiers (VCIs), where a virtual path (VP) is a bundle of virtual channels (VC). Traffic can be switched at the VP or VC level cell by cell. Associated with a VP or a VC is a quality of service (QoS) contract. In order to guarantee the switching of cells according to the contract without unacceptable cell loss, a number of queues are used at the input ports and output ports of the switch. Between input and output ports (including buffer queues and cell multiplexers and demultiplexers), a space matrix often is used, as in Figure 8.

Other switching techniques used in a telecommunication exchange include Ethernet and Rapid IO switches as well as IP packet routers, each with their own characteristics. Embedded IP routers, for IP forwarding, are useful for nodes that connect different IP networks. Sometimes, several switches are used inside a node.

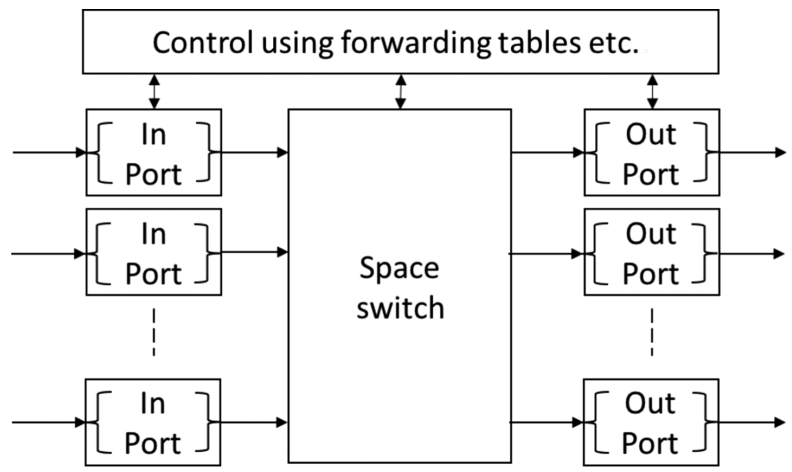

Figure 8. Typical ATM or packet router/switch structure with input and output buffering ports and control space switch. 


\section{QUALITY OF SERVICE REQUIREMENTS}

\subsection{Availability}

High availability of the telecom network and associated services is important. The requirements on maximum unavailability are in the order of one or a few minutes of subscriber unavailability per year. This includes downtime due to faults in the exchange or in the transmission equipment, but also unavailability due to faults in the software or due to planned software upgrades and often also accidents outside the control of the vendor, such as fires, damaged transmission cables, and incorrect operation of the exchange. Several methods are used to increase the availability of the exchange to the subscriber: redundancy, including fault-tolerant processors; segmentation; diagnostics of hardware and software faults; recovery after failure; handling of overload; disturbance-free upgrades and corrections; robustness to operator errors.

In order to cope with hardware faults redundancy, for example, redundant hardware is used for those parts of the switch that are critical for traffic execution. Requirements are in the order of 1000 years for the mean time between system failures (MTBSF). Often a redundant processor is available in synchronized execution (hot standby), ready for a transparent takeover if a single hardware fault occurs in one processor. An intelligent fault analysis algorithm is often used to decide which processor is faulty. In a multiprocessor system, $n+1$ redundancy is normally used, where each processor can be made of single, double, or triple hardware. When one processor fails, its tasks are moved to the idle (cool standby) processor. A similar redundancy method is based on load sharing, where the tasks of the failed processor are taken over by several of the other processors that are not overloaded themselves.

The group switch hardware is also normally duplicated or triplicated, because it is so vital to the exchange functions. The less central hardware devices, such as trunk devices, voice machines, transceivers, signal terminals, and code receivers, are normally pooled, so that a faulty device is blocked from use and all users can instead access the remaining devices until the faulty device is repaired.

To avoid system failure, a fault must be kept isolated within a small area of the exchange. This is done by segmentation of the hardware, with error supervision at the interfaces. In software, the segmentation is made by partitioning of and restricted access to data structures; only owners of data can change the data, where the owner can be a call process or a function.

After the occurrence of a fault in hardware or software, the fault must be identified, localized, and diagnosed, its effect restricted, and the exchange continue in its normal state of execution. For this to work, the fault diagnostics must be extensive and automatic. The exchange must be able to identify the faulty software and hardware and must be able to issue an alarm, usually to a remotely located operator.

After that a fault has been detected, the effect should be restricted to only the individual call or process (for instance, an operator procedure or a location update by a mobile subscriber) or an individual hardware device. This faulty call or process be aborted, while the rest of the exchange is not affected. This recovery process must be automatic and secure. In a small fraction of events, the fault may remain after the low-level recovery, or the initial fault is considered too severe by the fault handling software, so that a more powerful recovery procedure must be used. The process abort can be escalated to temporary blocking of hardware devices or software applications and, if required, result in the restart of an entire processor or a number of processors. If the restart fails in recovering the exchange into normal traffic handling, new data and software are loaded from internal or external memory and the exchange is then restarted.

An exchange is required to execute traffic literally nonstop and when overloaded by more traffic than it can handle, the overload handling including rejection of overflow traffic must be made gracefully. ITU requires, that an exchange that is offered $150 \%$ of what it was designed for should still have $90 \%$ of its maximum traffic handling capacity. The exchange must also be able to function without failure during extreme traffic loads. Such extreme loads can be both short peaks lasting a few milliseconds or sustained overload due to failures in other parts of the network. Overload handling is made by rejecting excess traffic very early in the call setup, before it has used too much processor time or scarce resources in the switching path. Figure 9 shows the overload performance with and without an overload control function.

Disturbance free upgrading of software packages and replacement or extension of hardware to the exchange should be supported ensure that ongoing traffic execution is not harmed. This both for fault corrections and when new functions are introduced. The architecture must thus allow traffic to be executed in redundant parts, while some parts of the exchange are upgraded.

Security against unauthorized access is implemented by use of passwords and physically locked exchange premises. To support robustness in case of operator errors, the user interface part of the exchange supervise that valid operation instructions are followed for operation and maintenance, or else issue an alert or prohibit other procedures. Logging of operator procedures and functions for undoing a procedure can be used. If a circuit board or other hardware part is incorrectly removed from the exchange, the

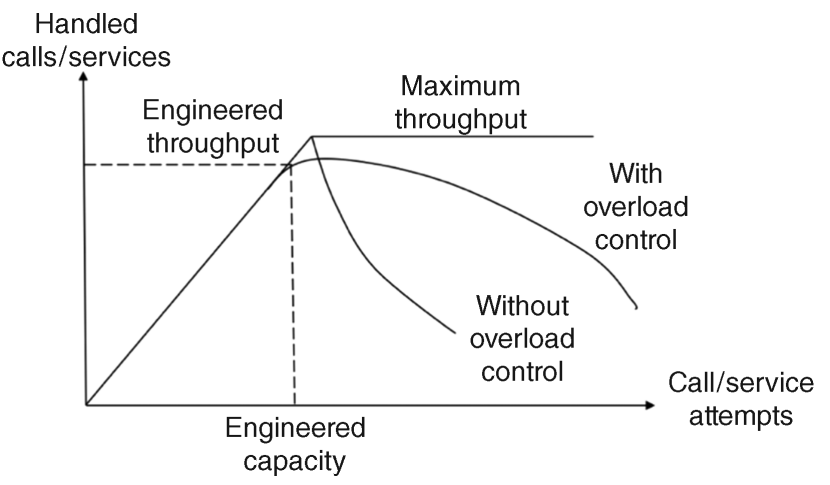

Figure 9. Call capacity as function of traffic load (number of call attempts) with and without overload control. 
exchange should restrict the fault to that particular part, use redundant hardware to minimize the effect of the fault, and indicate that this part is unavailable.

The real-time delays in the exchange must be restricted to transmit speech correctly, to ensure grade of service requirements. Highly loaded packet-switched connections may have problems achieving good real-time speech quality for this reason. Circuit-switched networks have better real-time performance regarding delays and grade of service for voice and video, compared with packet data networks. ATM switching, due to its short and fixed size cell/ packets, also fulfills the requirements. However, due to higher and higher bandwidths and capacity but also due to the use of label switching (virtual circuit) techniques, packet switching now usually give good quality of service and this even for real-time video.

\subsection{Scalability}

There is a need for scalable exchanges regarding capacity, from very small (such as local exchanges in desolate areas) to very large exchanges needed in dense metropolitan areas, transit exchanges, and MSCs. Furthermore, there is sometimes also a need for downward scalability regarding physical size and power consumption, particularly for indoor or inner city mobile telephony.

The upward scalability is limited by dynamic real-time call setup capacity; static call traffic handling capacity; grade of service (delays); memory limits; data transfer capacity; and dependability risks.

The downward scalability is limited by the cost to manufacture, operate, and handle; limited gain due to sharing of resources; physical size is limited by requirements for robustness; and energy consumption limited by the hardware technology available.

Processing Capacity. Advanced services and techniques require more processing capacity. This trend has been valid for the replacement of analog technology with digital processor-controlled functions and also the development of signaling systems from decadic via multifrequency to packet mode digital signaling, including trunk signaling protocols such as ISDN user part (ISUP) together with mobile application part (MAP) and transaction capability application part (TCAP) and the handling of mobility. In the charging area, going from pulse metering, via detailed billing and now toward flat rate also affects the call capacity. The number of calls per subscriber has increased due to lower call costs from deregulation and due to the use of subscriber redirection and answering services. Users has also changed their behavior due being more available via their mobile phones usually resulting in more but shorter calls.

Traffic Capacity. It is very important ensure sufficiently low congestion in the exchange and in the network. Normally, the switch fabric is virtually nonblocking, and the congestion occurs in other resources, such as access lines, trunk lines, and other equipment. The relation between congestion probability and the amount of traffic is well known, if all devices are accessible and the traffic follows a
Poisson process; that is, the times between offered calls are independent and exponentially distributed. In these cases, the probability can be calculated. In more complex traffic scenarios with non-Poisson traffic, the congestion probabilities are analyzed by help of simulation techniques.

Memory. The amount of memory used per subscriber line or trunk line is a way to measure the complexity of a telecom application. The trend in this area is similar to that of processing capacity, and the same factors are responsible for the increase in memory needs. Due to the real-time requirements, fast primary memory is used extensively, and secondary memory is only used for storage of backups, $\log$ files, and other data where time is not critical.

Transfer Capacity. A third part of the switching system's capacity is the data transfer from the exchange to other nodes, for example, to other exchanges and network databases, billing centers, statistical post processing, and nodes for centralized operation. There has been a growing demand for signaling link capacity due to large STPs, for transfer capacity from the exchange to a billing center due to detailed billing and large amounts of real-time statistics, and to transfer capacity into the exchange due to the increased amount of memory to reload at exchange failure.

Dependability Risks. Although dependability has increased for digital exchanges, there is a limit as to how large the nodes in the network that can be built. The more hardware and the more software functions assembled in one exchange, the more errors there are. The vast majority of these faults will be handled by low-level recovery, transparent to the telecom function, or only affecting one process. However, a small fraction of the faults can result in a major outage that affects the entire exchange during some time. The risk of a 1-h complete exchange failure during a year for one exchange must be low. If we add the functionality of an exchange node we increase the amount of software and presumably the number of faults in the node; the risk of a major outage should be larger; and especially in a new exchange introducing new software, potentially with new faults. Unavailability due to completely stopped traffic execution must be much less than the total effect of software process abortions and devices blocked due to hardware errors. Another argument for limiting the size and complexity of the nodes from a dependability point of view is that network redundancy is then required but can only be used if there are several exchanges in the network.

\subsection{Life-Cycle Cost}

Since the 1980 s, life-cycle-related costs such as the operating cost has become larger than the investment cost of an exchange. Life-cycle costs are also due to product handling matters, such as ordering, installation, and spare part supply. The emphasis on more efficient operation and maintenance has increased, regarding both ease of use and utilization of centers that remotely operate and maintain a number of exchanges that are not staffed.

For central operation, more robust methods for remote activities have evolved. Software upgrades and corrections, 
alarm supervision and handling, collection of statistics and charging data, and handling and definition of subscriber data are all made remotely. Transmission uses a multitude of techniques and protocols. Open standard protocols have taken over from proprietary protocols. For ease of use, the telecommunication management network (TMN) was an attempt by ITU to standardize the operator interface. After several years, this standard is still not used much. Instead, the operator interface is to a large extent dependent on the exchange manufacturer as well as the requirements from the telecom operator company. The coexistence of several open and proprietary user interfaces is common.

\section{EVOLUTION TRENDS}

\subsection{Technology Trends}

Semiconductor process improvements, higher chip fabrication yields, and increasing numbers of connectivity layers result in that the storage capability of memories and the execution speed of processors have doubled every 18 months during almost 50 years, following the so called Moore's law. This exponential growth of transistors per chip has now slowed down somewhat due to voltage limits, current leaks, clock frequency, and power density limits; and enforce new more parallel hardware architectures in order to keep energy use within reasonable limits.

The development of optical fibers, including the introduction of wavelength multiplexing, has also evolved very fast. There are still many factors that lead to cheaper nodes and higher bit rates in the network. At the same time, digital coding and compression techniques have been improved in an almost equally impressing rate that makes it possible to transmit voice with traditional telecom quality using only a fraction of the bandwidth that was used yesterday. Video services making use of much larger bandwidths have also become common. These developments are changing the design of both nodes and networks.

It is also important to provide increased interoperability between network standards, since the end users do not want to be concerned about where a person is physically located or to which network the person is connected. The introduction of universal personal numbers solves this and has lead to a convergence of fixed and mobile telephony. The possibility of accessing short message services (SMS), fax, and e-mail via both fixed and mobile devices and voice over IP (VOIP) and Video on Demand are examples of services that illustrate the needs for interoperability and convergence of the tele- and data-communication networks. A problem today seems to be that most proprietary IPbased voice and messaging services requires their users to create new personal user IDs in a way controlled by the service providers. You may at best be able to use your (Google, Apple, Facebook, etc.) ID for more than one service and sometimes also in other systems.

High bit rates at a low price, combined with the demand for real-time multimedia services, indicate that the network must either become more flexible or consist of several different but interoperating networks. Packet and new, more flexible circuit switching techniques supported by new protocols (such as MPLS) can solve these needs. In order to integrate service execution, control and connectivity horizontally across multiple access networks, a layered architecture approach can be used using a common transport layer based on IP and Ethernet technology over fiber rather than delivering single services such as voice telephony or data access (vertically integrated networks). This architecture supports efficient IP packet-based transport of both signaling and payload. By using a common mostly IPbased infrastructure one can handle all network services for communication between fixed and mobile terminals.

\subsection{Network Convergence and Evolution}

Calls and other similar media sessions can now be setup in many ways and the protocols and messages used have changed over the years. The ISDN call setup protocol mentioned previously has been complemented with protocol collections such as H.323 that enable call setup including coded and compressed voice calls and the choice of what coding standard to use for a specific call session. The Session Initiation Protocol (SIP) has a similar but more limited scope for the setup of communication sessions between two parties and selection of coding standard using the Session Description Protocol (SDP).

The IP Multimedia Subsystem (IMS) architecture defined by the European Telecommunications Standards Institute (ETSI) and by the 3rd-Generation Partnership Project (3GPP) allow video and other media forms to be exchanged and charged on a session-by-session basis from peer to peer in a way similar to classic phone calls. The IMS architecture uses a call session control function (CSCF) (important part of an IMS application module) as handler of SIP-initiated sessions.

The Internet and the possibility to encapsulate and transport digitized and encoded voice packets over IP networks, refereed to as Voice over IP (VOIP), has changed the world of telecommunication a lot. Skype is one example of a popular service that allows voice calls to be setup between computers and other (computer containing) terminals such as smart phones. It also allows calls from a computer to any ordinary phone via a call server and media gateway connected to the phone network.

When computers are used as terminals for real-time communication a problem is that most users do not have their computers on all the time. A consequence is that you have to agree on some call setup conventions, like "you can mostly reach me between 18:00 and 20:00" or you need to announce your intention to setup a call by some other means like by sending an SMS. The problem has now more or less disappeared when most people use smart phones that are kept on most of the time. This means that people are reachable where and when they want.

The convergence and evolution toward a common infrastructure is sometimes called the New Generation Network (NGN) architecture (Figure 9), but also can be associated with several other concepts discussed below (Figure 10).

This network evolution extending beyond but perhaps inspired by IN is supported by techniques for separation of control and switching such as the protocol H.248 aimed to allow a so called soft switch to control one or more 


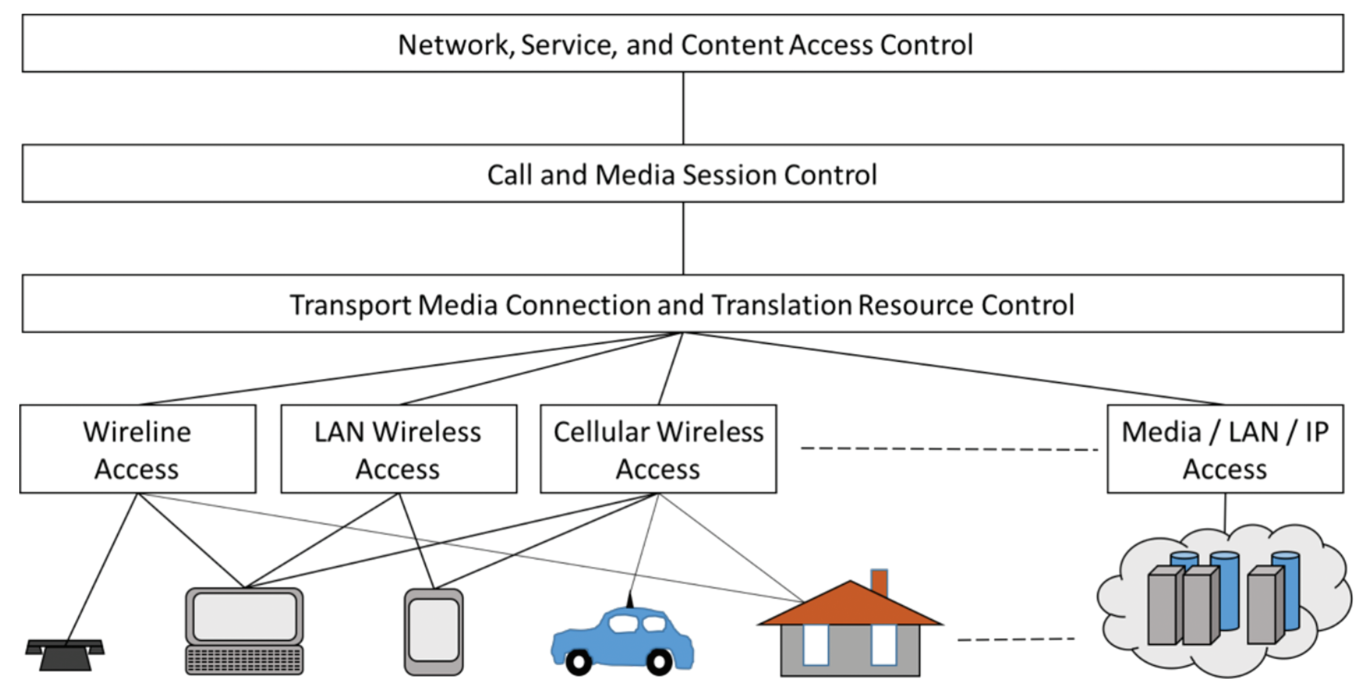

Figure 10. Converged network architecture with virtualized decoupling of access, transport, control, and service functions.

media gateways and/or switch nodes. Software-defined exchanges, soft-switches, or media gateway controllers include a call session control function (CSCF) for handling of voice calls and other session-oriented services. They are also responsible for that sessions can be connected via a physical switch and media gateway (MGW) and handle the signaling between network nodes and other networks. The CSCF establishes a call session, and further manages reserved connection path resources, end to end. This for example through a backbone network based on an ATM, IP, or Ethernet and processors for media stream processing. The MGW nodes provides physical switching, coding translations, and interfaces to access nodes and other networks.

The software-defined network (SDN) is a similar virtualization approach that has evolved from the IP networking community. SDN proposes a logical or virtual network built as a layer above the physical concrete networking layers. It is intended to support a separation between control and data switching layers similar to ambitions such as SS7, IN, and NGN. More specifically using the OSI stack terminology a SDN is operating above and thus decouples from lower network layers such as the IP layer. The idea is to allow different services and users to share underlying hardware resources in a flexible and secure way. As in many other networking techniques this requires that new protocols are introduced to create, encapsulate, differentiate, and manage the networks, services, and users from each other. The protocols needed to establish and run SDN can essentially be separated in service setup protocols and transport protocols.

The behavior of a SDN is supposed to be setup using the OpenFlow protocol. OpenFlow allows a table driven and thus software-defined network behavior where not only the forwarding but also simple alteration actions of packet headers can be setup by a central network controller acting from above. Setting up what in essence is a virtual circuit connection speeding up the packet carried data flow.
Network function virtualization (NFV) is another architecture that is strongly related to SDN. NFV aims at making functions or services implemented as virtual network functions (VNF) that controls and gives services to the network to be as platform technology and location independent as possible. To achieve this, the VNF are intended to execute on one or more virtual machines in computing clusters or clouds, based on standardized memory and processing resources, made accessible via the network.

3GPP has been strongly influential on the development of $3 \mathrm{G}$ via the long-term evolution (LTE) plan toward $4 \mathrm{G}$ and now more recently towards $5 \mathrm{G}$, by ITU referred to as IMT2020. These last developments of the wireless technologies not only aim at much higher bandwidths but are for example also aimed to support very low energy connections to the so called Internet of Things (IoT) as well as device and device-to-device communications, thus supporting machine to machine (M2M) and vehicle to vehicle or vehicle to infrastructure (V2X) and connected cars and cooperative intelligent transport system (C-ITS) needs.

\section{BIBLIOGRAPHY}

1. R. L. Freeman. Telecommunication System Engineering, 4th ed.; Wiley, 2004.

2. D. Minoli. Telecommunications Technology Handbook. Artech House, 1991.

3. M. Schwartz. Telecommunication Networks: Protocols, Modelling and Analysis. Addison-Wesley, 1987.

TONY LaRsson

Halmstad University, Halmstad, Sweden 